\title{
Symmetry Breaking in the Static Coordinate System of de Sitter Spacetime
}

\author{
András Kaiser ${ }^{1}$ and Alan Chodos ${ }^{2}$ \\ Center for Theoretical Physics, Sloane Physics Laboratory \\ Yale University, New Haven, Connecticut 06520-8120
}

\footnotetext{
${ }^{1}$ andras@genesis2.physics.yale.edu

2 chodos@yalph2.physics.yale.edu
} 


\begin{abstract}
:
We study symmetry breaking in the static coordinate-system of de Sitter space. This is done with the help of the functional-Schrödinger approach used in previous calculations by Ratra [1]. We consider a massless, minimally coupled scalar field as the parameter of a continuous symmetry (the angular component of an $\mathrm{O}(2)$ symmetry). Then we study the correlation function of the massless scalar field, to derive the correlation function of the original field, which finally shows the restoration of the continuous symmetry.

\section{Introduction}

Studying quantum field theory in de Sitter space has a long history [2], and the interest in the inflationary model of the universe brought some attention to the study of symmetry breaking in this spacetime [3]. As is known from previous results $[1,4]$, the question of whether an internal symmetry is spontaneously broken in a field theory quantized on a de Sitter background may depend on the coordinate system that is chosen. In this paper, we choose to do the computation for a minimally coupled scalar field in the so-called static coordinate system, which is distinguished by the fact that the interpretation of the results is particularly transparent in this case.

The coordinate-system we wish to use has the special property that its spatial sections are geodesic hypersurfaces with identical metric, and the time coordinate for each slice is given by the proper time of the observer whose spatial coordinate is the origin. In this sense this system is the one that describes the spacetime as a geodesic observer perceives it. It inherently excludes anything beyond the event horizon of the observer, and manifests time-translational invariance, because time-translation is generated by an element of the de Sitter group (which is not the case for the systems used previously), namely the one that translates the observer along its world-line $\left(\Omega_{04}\right.$, rotation in the $0-4$ plane according to the coordinate definitions of section 2). Only if the field is quantized in this system, will the field modes have $e^{i \omega t}$ time-dependence, with the $t$ coordinate time being the proper time of the observer at the origin. The important consequence of this property is that this quantization gives us the Fock-space as perceived by the observer (i.e. the vacuum state of this quantization will be the state in which the observer doesn't detect particles, etc.). So if we want to define symmetry breaking according to the field measurements of our geodesic observer in its vacuum state, then we must calculate the expectation value of the field on the vacuum state of this quantization.
\end{abstract}

\title{
2.The static system
}

De Sitter spacetime can be embedded in a 5 D Minkowski-space (we use signature $<+,-,-,-,->$ ), where it is a $4 \mathrm{D}$ hypersurface determined by

$$
x^{02}-x^{12}-x^{2}-x^{32}-x^{2}=-\alpha^{2}
$$

where $\alpha$ is the de Sitter radius, which also determines the spacetime curvature, $R=12 \alpha^{-2}$ [5].

For the static system we will use a time and polar spatial coordinates, $t, r, \vartheta, \varphi$. Choosing the worldline of our geodesic observer to be

$$
x=(\alpha \sinh (t / \alpha), 0,0,0, \alpha \cosh (t / \alpha))
$$

in terms of the coordinates of the embedding space determines the geodesic 3-surfaces orthogonal to the worldline at every $t$, which are given by the $x^{0}=\tanh (t / \alpha) x^{4}$ condition within the de Sitter manifold. These spatial hypersurfaces meet on the 2 -surface

$$
x^{0}=0, \quad x^{12}+x^{2}+x^{2}=\alpha^{2}, \quad x^{4}=0
$$

This 2-surface thus forms a coordinate singularity in the static system, which must be exluded from the range of the coordinates. The physical reason for the existence of this singularity is that null-geodesics passing through it form the event horizon of our observer. We will use

$$
r^{2}=x^{1^{2}}+x^{2^{2}}+x^{3^{2}}
$$

radial coordinate on the geodesic 3 -surface of a time-instant, even though this is not the proper distance from the origin on the spatial sections. The proper distance from the origin on these hypersurfaces is given 
by $s=\alpha \arcsin (r / \alpha)$. We also introduce polar coordinates on the $r=$ const. $t=$ const. 2 -spheres in the usual way.

The embedding relations of the static coordinate system are given by:

$$
\begin{aligned}
& x^{0}=\alpha\left(1-r^{2} / \alpha^{2}\right)^{1 / 2} \sinh (t / \alpha) \\
& x^{1}=r \sin \vartheta \sin \varphi \\
& x^{2}=r \sin \vartheta \cos \varphi \\
& x^{3}=r \cos \vartheta \\
& x^{4}=\alpha\left(1-r^{2} / \alpha^{2}\right)^{1 / 2} \cosh (t / \alpha)
\end{aligned}
$$

where the coordinate ranges are:

$$
t:(-\infty, \infty), \quad r:[0, \alpha), \quad \vartheta:[0, \pi], \quad \varphi:[0,2 \pi]
$$

As we can see from these relations, the static system covers only a strip on the de Sitter manifold, for which $x^{1^{2}}+x^{2}+x^{3}<\alpha^{2}$. This is the set of points on the manifold, which are in causal relation with the observer in both directions.

The metric in this coordinate system is given by:

$$
d s^{2}=\left(1-r^{2} / \alpha^{2}\right) d t^{2}-\frac{1}{1-r^{2} / \alpha^{2}} d r^{2}-r^{2}\left\{d \vartheta^{2}+\sin ^{2} \vartheta d \varphi^{2}\right\}
$$

from which we can see that the observer at the origin perceives an effect of time dilatation, i.e. time seems to pass slower as one looks further away, an effect due to the spacetime curvature (similarly to decreasing radial coordinate near a black hole).

We write the action for a minimally coupled scalar field $\phi$, with mass m:

$$
S=\int d t d r d \vartheta d \varphi r^{2} \sin \vartheta\left\{\frac{\frac{1}{2}|\dot{\phi}|^{2}}{1-r^{2} / \alpha^{2}}-\left(1-r^{2} / \alpha^{2}\right) \frac{1}{2}\left|\frac{\partial \phi}{\partial r}\right|^{2}+\frac{1}{2} \frac{\phi^{*} \mathcal{L}_{\vartheta \varphi}(\phi)}{r^{2}}-\frac{m^{2}}{2}|\phi|^{2}\right\}
$$

where $\mathcal{L}_{\vartheta \varphi}$ is the angular part of the Laplacian at unit radius:

$$
\mathcal{L}_{\vartheta \varphi}=\frac{\partial^{2}}{\partial \vartheta^{2}}+\cot \vartheta \frac{\partial}{\partial \vartheta}+\frac{1}{\sin ^{2} \vartheta} \frac{\partial^{2}}{\partial \varphi^{2}}
$$

This action leads to the wave equation:

$$
0=\frac{r^{2}}{1-r^{2} / \alpha^{2}} \frac{\partial^{2} \phi}{\partial t^{2}}-\frac{\partial}{\partial r}\left(r^{2}\left(1-r^{2} / \alpha^{2}\right) \frac{\partial \phi}{\partial r}\right)-\mathcal{L}_{\vartheta \varphi}(\phi)+r^{2} m^{2} \phi
$$

Notice that by redefining of the radial coordinate

$$
\begin{array}{cr}
\tilde{r}=\frac{1}{2} \alpha \ln \left(\frac{\alpha+r}{\alpha-r}\right) & r=\alpha \tanh (\tilde{r} / \alpha) \\
d \tilde{r}=\frac{d r}{1-r^{2} / \alpha^{2}} & d r=\frac{d \tilde{r}}{\cosh ^{2}(\tilde{r} / \alpha)} \\
\tilde{r}:[0, \infty) & r:[0, \alpha)
\end{array}
$$

we can bring the action to the following form:

$S=\int d t d \tilde{r} d \vartheta d \varphi(\alpha \tanh (\tilde{r} / \alpha))^{2} \sin \vartheta\left\{\frac{1}{2}|\dot{\phi}|^{2}-\frac{1}{2}\left|\frac{\partial \phi}{\partial \tilde{r}}\right|^{2}+\frac{1}{2 \cosh ^{2}(\tilde{r} / \alpha)} \frac{\phi^{*} \mathcal{L}_{\vartheta \varphi}(\phi)}{(\alpha \tanh (\tilde{r} / \alpha))^{2}}-\frac{m^{2}}{2 \cosh ^{2}(\tilde{r} / \alpha)}|\phi|^{2}\right\}$

and the wave equation: 


$$
0=(\alpha \tanh (\tilde{r} / \alpha))^{2} \frac{\partial^{2} \phi}{\partial t^{2}}-\frac{\partial}{\partial \tilde{r}}\left((\alpha \tanh (\tilde{r} / \alpha))^{2} \frac{\partial \phi}{\partial \tilde{r}}\right)-\frac{\mathcal{L}_{\vartheta \varphi}(\phi)}{\cosh ^{2}(\tilde{r} / \alpha)}+\frac{m^{2} \phi}{\cosh ^{2}(\tilde{r} / \alpha)}
$$

For $\tilde{r} / \alpha \gg 1$ the action becomes

$$
S=\int d t d \tilde{r} d \vartheta d \varphi \alpha^{2} \sin \vartheta\left\{\frac{1}{2}|\dot{\phi}|^{2}-\frac{1}{2}\left|\frac{\partial \phi}{\partial \tilde{r}}\right|^{2}\right\}
$$

and the wave equation

$$
0=\alpha^{2} \frac{\partial^{2} \phi}{\partial t^{2}}-\frac{\partial}{\partial \tilde{r}}\left(\alpha^{2} \frac{\partial \phi}{\partial \tilde{r}}\right)=\alpha^{2}\left(\frac{\partial^{2} \phi}{\partial t^{2}}-\frac{\partial^{2} \phi}{\partial \tilde{r}^{2}}\right)
$$

which yields plane waves with respect to the $\tilde{r}$ conformal radial coordinate in this limit. Looking at the relation between $r$ and $\tilde{r}$ again, we can check that the conformal coordinate of a point is given by the coordinate time that is needed for a light ray to travel between the origin and the point in question. From this we can also see that the plane wave gets infinitely compressed $(d \tilde{r} / d r \rightarrow \infty)$ as it approaches the event horizon, which is due to the time dilatation discussed previously, i.e. it takes infinite coordinate time for a light ray to reach the event horizon.

Now return to the original (non-conformal) radial parameter $r$, and look for solutions in the form:

$$
\Phi=e^{-i \omega t} \hat{\phi}_{\omega l m}(r, \vartheta, \varphi)=e^{-i \omega t} f_{\omega l}(r) Y_{l}^{m}(\vartheta, \varphi)
$$

The solutions are [6]:

$$
\begin{aligned}
& \hat{\phi}_{\omega l m}(r, \vartheta, \varphi)= \\
& =\frac{\Gamma\left(\frac{l}{2}+\frac{3}{4}+\frac{i \omega \alpha}{2}+\frac{1}{2} \sqrt{\frac{9}{4}-m^{2} \alpha^{2}}\right) \Gamma\left(\frac{l}{2}+\frac{3}{4}+\frac{i \omega \alpha}{2}-\frac{1}{2} \sqrt{\frac{9}{4}-m^{2} \alpha^{2}}\right)}{\sqrt{2 \pi} \Gamma\left(l+\frac{3}{2}\right) \Gamma(i \omega \alpha)}\left(\frac{r^{2}}{\alpha^{2}}\right)^{l / 2}\left(1-r^{2} / \alpha^{2}\right)^{\frac{i \omega \alpha}{2}} \\
& F\left(\frac{l}{2}+\frac{3}{4}+\frac{i \omega \alpha}{2}+\frac{1}{2} \sqrt{\frac{9}{4}-m^{2} \alpha^{2}}, \frac{l}{2}+\frac{3}{4}+\frac{i \omega \alpha}{2}-\frac{1}{2} \sqrt{\frac{9}{4}-m^{2} \alpha^{2}} ; l+\frac{3}{2} ; \frac{r^{2}}{\alpha^{2}}\right) Y_{l}^{m}(\vartheta, \varphi)
\end{aligned}
$$

where $F$ is the hypergeometric function. The solutions are orthonormalized with the following measure:

$$
\int d r d \vartheta d \varphi \frac{r^{2} \sin (\vartheta)}{1-r^{2} / \alpha^{2}} \hat{\phi}_{\omega^{\prime} l^{\prime} m^{\prime}}^{*}(r, \vartheta, \varphi) \hat{\phi}_{\omega l m}(r, \vartheta, \varphi)=\delta\left(\omega^{\prime}, \omega\right) \delta_{l^{\prime}, l} \delta_{m^{\prime}, m}
$$

We can expand the field in terms of these modes:

$$
\phi(r, \vartheta, \varphi)=\sum_{l, m} \int d \omega \xi_{\omega l m} \hat{\phi}_{\omega l m}(r, \vartheta, \varphi)
$$

then express the action:

$$
S=\sum_{l, m} \int d t d \omega\left\{\frac{1}{2}\left|\dot{\xi}_{\omega l m}\right|^{2}-\frac{\omega^{2}}{2}\left|\xi_{\omega l m}\right|^{2}\right\}
$$

We have the action of a simple harmonic oscillator for each mode, which has the ground-state wavefunction: 


$$
\psi\left(\xi_{\omega l m}, t\right)=e^{-\frac{i \omega t}{2}}\left(\frac{\omega}{\pi}\right)^{\frac{1}{4}} e^{-\frac{\omega}{2} \xi^{2}}
$$

from which immediately follows:

$$
<\xi_{\omega l m}^{2}>=\frac{1}{2 \omega}
$$

\section{The correlation functions}

Using this, and the field expansion, we can easily express the equal-time 2-point function:

$$
<\phi\left(r^{\prime}, \vartheta^{\prime}, \varphi^{\prime}\right), \phi(r, \vartheta, \varphi)>=\sum_{l, m} \int \frac{d \omega}{2 \omega} \hat{\phi}_{\omega l m}^{*}\left(r^{\prime}, \vartheta^{\prime}, \varphi^{\prime}\right) \hat{\phi}_{\omega l m}(r, \vartheta, \varphi)
$$

If we set $r^{\prime}=0$, the $l \neq 0$ modes can be neglected, since they are zero at the origin. We don't have to care about $\vartheta$ or $\varphi$ either, because of the spherical symmetry of the $l=0$ modes.

$$
\begin{gathered}
<\phi(0), \phi(r)>=\int \frac{d \omega}{2 \omega} \hat{\phi}_{\omega 00}^{*}(0) \hat{\phi}_{\omega 00}(r)= \\
=\int \frac{d \omega}{2 \omega} \frac{\Gamma\left(\frac{3}{4}-\frac{i \omega \alpha}{2}+\frac{1}{2} \sqrt{\frac{9}{4}-m^{2} \alpha^{2}}\right) \Gamma\left(\frac{3}{4}-\frac{i \omega \alpha}{2}-\frac{1}{2} \sqrt{\frac{9}{4}-m^{2} \alpha^{2}}\right)}{\sqrt{2 \pi} \sqrt{4 \pi} \Gamma\left(\frac{3}{2}\right) \Gamma(-i \omega \alpha)} \\
\frac{\Gamma\left(\frac{3}{4}+\frac{i \omega \alpha}{2}+\frac{1}{2} \sqrt{\frac{9}{4}-m^{2} \alpha^{2}}\right) \Gamma\left(\frac{3}{4}+\frac{i \omega \alpha}{2}-\frac{1}{2} \sqrt{\frac{9}{4}-m^{2} \alpha^{2}}\right)}{\sqrt{2 \pi} \sqrt{4 \pi} \Gamma\left(\frac{3}{2}\right) \Gamma(i \omega \alpha)}\left(1-r^{2} / \alpha^{2}\right)^{\frac{i \omega \alpha}{2}} \\
F\left(\frac{3}{4}+\frac{i \omega \alpha}{2}+\frac{1}{2} \sqrt{\frac{9}{4}-m^{2} \alpha^{2}} ; \frac{3}{4}+\frac{i \omega \alpha}{2}-\frac{1}{2} \sqrt{\frac{9}{4}-m^{2} \alpha^{2}} ; \frac{3}{2} ; r^{2} / \alpha^{2}\right)
\end{gathered}
$$

Examine the $r=0$ case first. The hypergeometric function gives 1 at zero argument, and since $\Gamma(i y) \Gamma(-i y)=\frac{\pi}{y \sinh (\pi y)}$, we have an expression with a powerlike ultraviolet divergence. In the massless case we also have an infrared divergence, due to the square root cancelling the $3 / 4$ in the argument of the gamma function.

Now examine $r \rightarrow \alpha \quad(r=\alpha$ is the event horizon, as discussed in section 2). Use the following transformation of the hypergeometric function [10]:

$$
\begin{gathered}
F(a, b ; c ; z)= \\
\frac{\Gamma(c) \Gamma(c-a-b)}{\Gamma(c-a) \Gamma(c-b)} F(a, b ; a+b-c+1 ; 1-z)+ \\
+(1-z)^{c-a-b} \frac{\Gamma(c) \Gamma(a+b-c)}{\Gamma(a) \Gamma(b)} F(c-a, c-b ; c-a-b+1 ; 1-z)
\end{gathered}
$$

which gives:

$$
\begin{gathered}
<\phi(0), \phi(r)>= \\
=\int \frac{d \omega}{16 \omega \pi^{2}}\left\{\frac{\Gamma\left(\frac{3}{4}+\frac{i \omega \alpha}{2}+\frac{1}{2} \sqrt{\frac{9}{4}-m^{2} \alpha^{2}}\right) \Gamma\left(\frac{3}{4}+\frac{i \omega \alpha}{2}-\frac{1}{2} \sqrt{\frac{9}{4}-m^{2} \alpha^{2}}\right)}{\Gamma\left(\frac{3}{2}\right) \Gamma(i \omega \alpha)}\left(1-r^{2} / \alpha^{2}\right)^{\frac{i \omega \alpha}{2}}\right. \\
F\left(\frac{3}{4}+\frac{i \omega \alpha}{2}+\frac{1}{2} \sqrt{\frac{9}{4}-m^{2} \alpha^{2}}, \frac{3}{4}+\frac{i \omega \alpha}{2}-\frac{1}{2} \sqrt{\frac{9}{4}-m^{2} \alpha^{2}} ; i \omega \alpha+1 ; 1-r^{2} / \alpha^{2}\right)+
\end{gathered}
$$




$$
\begin{gathered}
+\frac{\Gamma\left(\frac{3}{4}-\frac{i \omega \alpha}{2}+\frac{1}{2} \sqrt{\frac{9}{4}-m^{2} \alpha^{2}}\right) \Gamma\left(\frac{3}{4}-\frac{i \omega \alpha}{2}-\frac{1}{2} \sqrt{\frac{9}{4}-m^{2} \alpha^{2}}\right)}{\Gamma\left(\frac{3}{2}\right) \Gamma(-i \omega \alpha)}\left(1-r^{2} / \alpha^{2}\right)^{\frac{-i \omega \alpha}{2}} \\
\left.F\left(\frac{3}{4}+\frac{-i \omega \alpha}{2}+\frac{1}{2} \sqrt{\frac{9}{4}-m^{2} \alpha^{2}} \quad, \frac{3}{4}+\frac{-i \omega \alpha}{2}-\frac{1}{2} \sqrt{\frac{9}{4}-m^{2} \alpha^{2}} ;-i \omega \alpha+1 ; 1-r^{2} / \alpha^{2}\right)\right\}
\end{gathered}
$$

As $r \rightarrow \alpha$, the arguments of the hypergeometric functions approach zero, therefore their values approach 1 , so we set them to 1 for the purposes of this discusssion. In the next section we will need to know the behaviour of some of these expressions near the $r=\alpha$ limit, having the freedom to choose the radial coordinate arbitrarily close to $\alpha$. In the following analysis we will have to make some approximations to be able to investigate certain properties of this expression. These approximations will cause some finite error in the result, but it will not be crucial for two reasons. The first reason is that we will study certain divergences in the obtained expressions, for which the finite error is irrelevant. The second reason is that the error can be made arbitrarily small by choosing the radial coordinate sufficiently close to $\alpha$, when we evaluate the expressions.

On the other hand, the $\left(1-r^{2} / \alpha^{2}\right)^{ \pm \frac{i \omega \alpha}{2}} \quad$ term can be expressed as $e^{ \pm i \ln \left(1-r^{2} / \alpha^{2}\right) \frac{\omega \alpha}{2}}$, which results in an infinitely fast oscillation of this term with respect to $\omega$, as $r \rightarrow \alpha$.

Focus on the $m \rightarrow 0$ case, taking the $m \rightarrow 0$ limit before the $r \rightarrow \alpha$ limit, because this is the case that we will need in the next section. As $m \rightarrow 0$, we can substitute $\Gamma\left(\frac{3}{2}-\frac{m^{2} \alpha^{2}}{6} \pm \frac{i \omega \alpha}{2}\right)$ for $\Gamma\left(\frac{3}{4} \pm \frac{i \omega \alpha}{2}+\frac{1}{2} \sqrt{\frac{9}{4}-m^{2} \alpha^{2}}\right)$, and $\Gamma\left(\frac{m^{2} \alpha^{2}}{6} \pm \frac{i \omega \alpha}{2}\right) \quad$ for $\quad \Gamma\left(\frac{3}{4} \pm \frac{i \omega \alpha}{2}-\frac{1}{2} \sqrt{\frac{9}{4}-m^{2} \alpha^{2}}\right)$. As we take the $m \rightarrow 0$ limit, the error resulting from this substitution will also go to zero.

After these substitutions we have:

$$
\begin{gathered}
\int \frac{d \omega}{16 \omega \pi^{2}}\left\{\frac{\Gamma\left(\frac{3}{2}-\frac{m^{2} \alpha^{2}}{6}+\frac{i \omega \alpha}{2}\right) \Gamma\left(\frac{m^{2} \alpha^{2}}{6}+\frac{i \omega \alpha}{2}\right)}{\Gamma\left(\frac{3}{2}\right) \Gamma(i \omega \alpha)}\left(1-r^{2} / \alpha^{2}\right)^{\frac{i \omega \alpha}{2}}+\right. \\
\left.+\frac{\Gamma\left(\frac{3}{2}-\frac{m^{2} \alpha^{2}}{6}-\frac{i \omega \alpha}{2}\right) \Gamma\left(\frac{m^{2} \alpha^{2}}{6}-\frac{i \omega \alpha}{2}\right)}{\Gamma\left(\frac{3}{2}\right) \Gamma(-i \omega \alpha)}\left(1-r^{2} / \alpha^{2}\right)^{\frac{-i \omega \alpha}{2}}\right\}
\end{gathered}
$$

Now break up the $\omega$ integral into three parts:

$$
\begin{aligned}
0 & -m^{2} \alpha / 3 \\
m^{2} \alpha / 3 & -2 \pi /\left(-\alpha \ln \left(1-r^{2} / \alpha^{2}\right)\right) \\
2 \pi /\left(-\alpha \ln \left(1-r^{2} / \alpha^{2}\right)\right) & -\infty
\end{aligned}
$$

Taking the $m \rightarrow 0$ limit faster than the $r \rightarrow \alpha$ limit assures this order and the $m^{2} \alpha / 3 \ll$ $2 \pi /\left(-\alpha \ln \left(1-r^{2} / \alpha^{2}\right)\right.$ condition.

Consider the first part. The above condition allows us to substitute 1 for $\left(1-r^{2} / \alpha^{2}\right)^{ \pm \frac{i \omega \alpha}{2}}$. As the $m \rightarrow 0$ limit is taken faster than the $r \rightarrow \alpha$ limit, $\omega \ln \left(1-r^{2} / \alpha^{2}\right)$ goes to zero, justifying the above approximation. Also, all the errors that the following approximations will cause go to zero with $m \rightarrow 0$. Substitute $\Gamma\left(\frac{3}{2}\right)$ for $\Gamma\left(\frac{3}{2}-\frac{m^{2} \alpha^{2}}{6} \pm \frac{i \omega \alpha}{2}\right)$, since $m \rightarrow 0$, and $\frac{\omega \alpha}{2} \leq \frac{m^{2} \alpha^{2}}{6}$ in this interval, and the gamma function does not have a pole at $3 / 2$. Then substitute $1 / z$ for $\Gamma(z)$, wherever $|z| \ll 1$, which gives us

$$
\int_{0}^{m^{2} \alpha / 3} \frac{d \omega}{16 \omega \pi^{2}}\left\{\frac{i \omega \alpha}{\frac{m^{2} \alpha^{2}}{6}+\frac{i \omega \alpha}{2}}+\frac{-i \omega \alpha}{\frac{m^{2} \alpha^{2}}{6}-\frac{i \omega \alpha}{2}}\right\}
$$

which shows that this first of the three parts goes to a (finite) constant as $m \rightarrow 0$.

Next consider the third interval of integration, $\omega: 2 \pi /\left(-\alpha \ln \left(1-r^{2} / \alpha^{2}\right)\right)-\infty$. Here we can replace $\Gamma\left(\frac{3}{2}-\frac{m^{2} \alpha^{2}}{6} \pm \frac{i \omega \alpha}{2}\right) \quad$ with $\Gamma\left(\frac{3}{2} \pm \frac{i \omega \alpha}{2}\right)$, and $\Gamma\left(\frac{m^{2} \alpha^{2}}{6} \pm \frac{i \omega \alpha}{2}\right) \quad$ with $\Gamma\left( \pm \frac{i \omega \alpha}{2}\right) \quad$ in the $m \rightarrow 0$ limit. Then use $\Gamma( \pm i \omega \alpha)=(2 \pi)^{-\frac{1}{2}} 2^{ \pm i \omega \alpha-\frac{1}{2}} \Gamma\left(\frac{1}{2} \pm \frac{i \omega \alpha}{2}\right) \Gamma\left( \pm \frac{i \omega \alpha}{2}\right)$ 
This way we get

$$
\begin{gathered}
\int_{2 \pi /\left(-\ln \left(1-r^{2} / \alpha^{2}\right)\right)}^{\infty} \frac{d \omega}{16 \omega \pi^{2}}\left\{\frac{\Gamma\left(\frac{3}{2}+\frac{i \omega \alpha}{2}\right) \Gamma\left(\frac{i \omega \alpha}{2}\right)}{\Gamma\left(\frac{3}{2}\right)(2 \pi)^{-\frac{1}{2}} 2^{i \omega \alpha-\frac{1}{2}} \Gamma\left(\frac{1}{2}+\frac{i \omega \alpha}{2}\right) \Gamma\left(\frac{i \omega \alpha}{2}\right)}\left(1-r^{2} / \alpha^{2}\right)^{\frac{i \omega \alpha}{2}}+\right. \\
\left.\quad+\frac{\Gamma\left(\frac{3}{2}-\frac{i \omega \alpha}{2}\right) \Gamma\left(\frac{-i \omega \alpha}{2}\right)}{\Gamma\left(\frac{3}{2}\right)(2 \pi)^{-\frac{1}{2}} 2^{-i \omega \alpha-\frac{1}{2}} \Gamma\left(\frac{1}{2}-\frac{i \omega \alpha}{2}\right) \Gamma\left(\frac{-i \omega \alpha}{2}\right)}\left(1-r^{2} / \alpha^{2}\right)^{\frac{-i \omega \alpha}{2}}\right\}
\end{gathered}
$$

then use $\Gamma(z+1)=z \Gamma(z)$, cancel the appropriate terms, and rearrange:

$$
\int_{2 \pi /\left(-\alpha \ln \left(1-r^{2} / \alpha^{2}\right)\right)}^{\infty} \frac{d \omega}{16 \omega \pi^{2}}\left\{\frac{(1+i \omega \alpha) \sqrt{\pi}}{\Gamma\left(\frac{3}{2}\right)}\left(\frac{1-r^{2} / \alpha^{2}}{4}\right)^{\frac{i \omega \alpha}{2}}+\frac{(1-i \omega \alpha) \sqrt{\pi}}{\Gamma\left(\frac{3}{2}\right)}\left(\frac{1-r^{2} / \alpha^{2}}{4}\right)^{\frac{-i \omega \alpha}{2}}\right\}
$$

break it into two integrals:

$$
\begin{gathered}
\int_{2 \pi /\left(-\alpha \ln \left(1-r^{2} / \alpha^{2}\right)\right)}^{\infty} \frac{d \omega}{16 \omega \pi^{2}}\left\{\frac{\sqrt{\pi}}{\Gamma\left(\frac{3}{2}\right)}\left(\frac{1-r^{2} / \alpha^{2}}{4}\right)^{\frac{i \omega \alpha}{2}}+\frac{\sqrt{\pi}}{\Gamma\left(\frac{3}{2}\right)}\left(\frac{1-r^{2} / \alpha^{2}}{4}\right)^{\frac{-i \omega \alpha}{2}}\right\}+ \\
+\int_{2 \pi /\left(-\alpha \ln \left(1-r^{2} / \alpha^{2}\right)\right)}^{\infty} \frac{d \omega}{16 \pi^{2}}\left\{\frac{i \alpha \sqrt{\pi}}{\Gamma\left(\frac{3}{2}\right)}\left(\frac{1-r^{2} / \alpha^{2}}{4}\right)^{\frac{i \omega \alpha}{2}}+\frac{-i \alpha \sqrt{\pi}}{\Gamma\left(\frac{3}{2}\right)}\left(\frac{1-r^{2} / \alpha^{2}}{4}\right)^{\frac{-i \omega \alpha}{2}}\right\}
\end{gathered}
$$

where the first integral is finite because of the following. As a consequence of the oscillation, the integral will consist of contributions with periodically alternating sign, and decreasing magnitude. Therefore the first contribution gives an upper bound to the integral. We get an upper bound to this first contribution, if we multiply the length of the interval (half period, $2 \pi /\left[\alpha\left(-\ln \frac{1-r^{2} / \alpha^{2}}{4}\right)\right]$ ), with the supremum of the function in the interval, which gives

$$
\frac{2 \pi}{\alpha\left(-\ln \frac{1-r^{2} / \alpha^{2}}{4}\right)} \frac{\sqrt{\pi} / \Gamma\left(\frac{3}{2}\right)}{16 \frac{2 \pi}{-\alpha \ln \left(1-r^{2} / \alpha^{2}\right)} \pi^{2}}=\frac{\Gamma\left(\frac{3}{2}\right)}{16 \pi \sqrt{\pi}} \frac{\ln \left(1-r^{2} / \alpha^{2}\right)}{\ln \left(1-r^{2} / \alpha^{2}\right)-\ln (4)}
$$

which is finite $(0<r<\alpha, r \rightarrow \alpha)$

The second integral is the integral of a periodic oscillating function, which we regulate with an $e^{-\epsilon \omega}$ type ultraviolet cutoff, then take $\epsilon \rightarrow 0$ limit, obtaining

$$
\begin{gathered}
\int_{2 \pi /\left(-\alpha \ln \left(1-r^{2} / \alpha^{2}\right)\right)}^{\infty} \frac{\alpha}{16 \pi \sqrt{\pi} \Gamma\left(\frac{3}{2}\right)}\left\{i e^{-i\left(\ln \left(\frac{4}{1-r^{2} / \alpha^{2}}\right) \frac{\omega \alpha}{2}-i \epsilon \omega\right)}-i e^{i\left(\ln \left(\frac{4}{1-r^{2} / \alpha^{2}}\right) \frac{\omega \alpha}{2}+i \epsilon \omega\right)}\right\}= \\
=\frac{\alpha}{16 \pi \sqrt{\pi} \Gamma\left(\frac{3}{2}\right)}\left[\frac{i}{-i\left(\ln \left(\frac{4}{1-r^{2} / \alpha^{2}}\right) \frac{\alpha}{2}-i \epsilon\right)} e^{-i\left(\ln \left(\frac{4}{1-r^{2} / \alpha^{2}}\right) \frac{\omega \alpha}{2}-i \epsilon \omega\right)}+\right. \\
\left.+\frac{-i}{i\left(\ln \left(\frac{4}{1-r^{2} / \alpha^{2}}\right) \frac{\alpha}{2}+i \epsilon\right)} e^{i\left(\ln \left(\frac{4}{1-r^{2} / \alpha^{2}}\right) \frac{\omega \alpha}{2}+i \epsilon \omega\right)}\right]_{2 \pi /\left(-\alpha \ln \left(1-r^{2} / \alpha^{2}\right)\right)}^{\infty} \\
=\frac{\alpha}{16 \pi \sqrt{\pi} \Gamma\left(\frac{3}{2}\right)}\left(\frac{1}{\ln \left(\frac{4}{1-r^{2} / \alpha^{2}}\right) \frac{\alpha}{2}-i \epsilon} e^{-i\left(\pi \frac{\ln \left(1-r^{2} / \alpha^{2}\right)-\ln (4)}{\ln \left(1-r^{2} / \alpha^{2}\right)}-\frac{i^{2} 2 \pi}{-\alpha \ln \left(1-r^{2} / \alpha^{2}\right)}\right)}+\right.
\end{gathered}
$$




$$
\left.+\frac{1}{\ln \left(\frac{4}{1-r^{2} / \alpha^{2}}\right) \frac{\alpha}{2}+i \epsilon} e^{i\left(\pi \frac{\ln \left(1-r^{2} / \alpha^{2}\right)-\ln (4)}{\ln \left(1-r^{2} / \alpha^{2}\right)}+\frac{i \epsilon 2 \pi}{-\alpha \ln \left(1-r^{2} / \alpha^{2}\right)}\right)}\right)
$$

which is, once again, finite, and in the $\epsilon \rightarrow 0$ limit yields

$$
\frac{1}{4 \pi \sqrt{\pi} \Gamma\left(\frac{3}{2}\right) \ln \left(\frac{4}{1-r^{2} / \alpha^{2}}\right)} \cos \left(\pi \frac{\ln \left(1-r^{2} / \alpha^{2}\right)-\ln (4)}{\ln \left(1-r^{2} / \alpha^{2}\right)}\right)
$$

from which we can see that this interval also gives a finite contribution to the total integral.

Now consider the second interval of integration, $\omega: m^{2} \alpha / 3-2 \pi /\left(-\alpha \ln \left(1-r^{2} / \alpha^{2}\right)\right)$. Here we can neglect the oscillation of the $\left(1-r^{2} / \alpha^{2}\right)^{ \pm \frac{i \omega \alpha}{2}} \quad$ term without changing the magnitude of the result, because the upper limit of integration was determined such that the phase change of this term always stays less than $\pi$. Also we can substitute $\Gamma\left(\frac{3}{2}\right)$ for $\Gamma\left(\frac{3}{2}-\frac{1}{6} m^{2} \alpha^{2} \pm \frac{i \omega \alpha}{2}\right)$, because $m^{2} \alpha / 3 \ll 2 \pi /\left(-\alpha \ln \left(1-r^{2} / \alpha^{2}\right)\right)$, and from $\quad r \approx \alpha$ follows $2 \pi /-\ln \left(1-r^{2} / \alpha^{2}\right) \ll 1$, so we have both $\quad \frac{m^{2} \alpha^{2}}{6} \ll 1$ and $\frac{\omega \alpha}{2} \ll 1$. From this also follows that we can use the $1 / z$ substitution for $\Gamma(z)$ for the $\Gamma\left(\frac{m^{2} \alpha^{2}}{6} \pm \frac{i \omega \alpha}{2}\right)$ and $\Gamma( \pm i \omega \alpha)$ terms.

As we see, we finally arrive at the same expression as in the first part, but here we have different limits for the integration.

$$
\begin{gathered}
\int_{m^{2} \alpha / 3}^{2 \pi /\left(-\alpha \ln \left(1-r^{2} / \alpha^{2}\right)\right)} \frac{d \omega}{16 \omega \pi^{2}}\left\{\frac{i \omega \alpha}{\frac{m^{2} \alpha^{2}}{6}+\frac{i \omega \alpha}{2}}+\frac{-i \omega \alpha}{\frac{m^{2} \alpha^{2}}{6}-\frac{i \omega \alpha}{2}}\right\} \\
=\int_{m^{2} \alpha / 3}^{2 \pi /\left(-\alpha \ln \left(1-r^{2} / \alpha^{2}\right)\right)} \frac{d \omega}{16 \omega \pi^{2}} \frac{(\omega \alpha)^{2}}{\left(\frac{m^{2} \alpha^{2}}{6}\right)^{2}+\left(\frac{\omega \alpha}{2}\right)^{2}} \\
=\left.\int_{\frac{1}{8 \pi^{2}}} \ln \left(\left(\frac{\omega \alpha}{2}\right)^{2}+\left(\frac{m^{2} \alpha^{2}}{6}\right)^{2}\right)\right|_{\omega=m^{2} \alpha / 3} ^{2 \pi /\left(-\alpha \ln \left(1-r^{2} / \alpha^{2}\right)\right)} \\
=\frac{1}{8 \pi^{2}} \frac{\alpha \frac{\omega \alpha}{2}}{\left(\frac{m^{2} \alpha^{2}}{6}\right)^{2}+\left(\frac{\omega \alpha}{2}\right)^{2}} \\
\ln \left(\frac{\left.\left(1-r^{2} / \alpha^{2}\right)\right)}{\ln \left(\frac{\pi}{-\ln \left(1-r^{2} / \alpha^{2}\right)}\right)^{2}+\left(\frac{m^{2} \alpha^{2}}{6}\right)^{2}}\right. \\
2\left(\frac{m^{2} \alpha^{2}}{6}\right)^{2}
\end{gathered}
$$

which is a divergent expression in the $m \rightarrow 0$ limit. We will need this expression in the next section.

\section{Symmetry breaking}

Now apply these results to the problem of symmetry breaking. Consider a model with complex scalar field $\Phi$ and a symmetry-breaking $\Phi^{4}$ potential. We will use the approximation of a fixed radial component of the complex scalar field at the classical minimum, $|\Phi|=\rho_{0}$, which leaves us with a massless minimally coupled scalar field as the angular component. We give a summary of this treatment. For a fuller discussion see $[1]$.

In a broken-symmetry vacuum state the $\langle\Phi(0) \Phi(r)>$ correlation does not go to zero as we let $r$ approach the event horizon, so a vanishing correlation is evidence for symmetry restoration. Express $<\Phi(0) \Phi(r)>$ with the angular field:

$$
<\Phi(0) \Phi(r)>=\rho_{0}^{2}<e^{-i \frac{\phi(\mathbf{0})}{\rho_{0}}} e^{i \frac{\phi(\mathbf{r})}{\rho_{0}}}>
$$


Divide the angular field operator into creation and annihilation parts, and commute the former to the left, the latter to the right, to annihilate the vacuum states at the respective ends. Then the remaining commutators give us:

$$
<\Phi(0) \Phi(r)>=\rho_{0}^{2} e^{<\frac{\phi(\mathbf{0}) \phi(\mathbf{r})}{\rho_{0}^{2}}>-<\frac{\phi(\mathbf{0}) \phi(\mathbf{0})}{\rho_{0}^{2}}>}
$$

which we will have to renormalize due to the ultraviolet divergence in the second term (the two terms of the exponent are also infrared divergent separately, but these divergences cancel each other, so we can take finite mass, calculate the exponent then let the mass go to zero). We can renormalize by dividing the whole expression by $<\Phi(0) \Phi\left(r_{0}\right)>$, where we keep $r_{0}$ at any fixed value while letting $r$ go to the event horizon.

$$
\frac{<\Phi(0) \Phi(r)>}{<\Phi(0) \Phi\left(r_{0}\right)>}=e^{<\frac{\phi(\mathbf{0}) \phi(\mathbf{r})}{\rho_{0}^{2}}>-<\frac{\phi(\mathbf{0}) \phi\left(\mathbf{r}_{\mathbf{0}}\right)}{\rho_{0}^{2}}>}
$$

As we have seen previously, for $r \rightarrow \alpha, \quad\langle\phi(0) \phi(r)\rangle$ is given by an infrared divergent integral (it diverges as we let the mass that regulates the infrared behaviour go to zero), which divergence also has a $-\ln \left(-\ln \left(1-r^{2} / \alpha^{2}\right)\right)$ dependence. From this follows, that as $r \rightarrow \alpha$, the exponent, $\left\langle\frac{\phi(0) \phi(r)}{\rho_{0}^{2}}>-\left\langle\frac{\phi(0) \phi\left(r_{0}\right)}{\rho_{0}^{2}}>\right.\right.$ will diverge as $-\ln \left(-\ln \left(1-r^{2} / \alpha^{2}\right)\right)$ in other words it will go to $-\infty$ - (It might be of interest to note, that in terms of the conformal radius $\tilde{r}$ this expression for the exponent takes the form (in the $r \rightarrow \alpha$ limit): $\quad-\ln (2 \tilde{r} / \alpha)$, a single logarithmic behaviour instead of a double.)

This means, that the $\frac{\langle\Phi(0) \Phi(r)>}{\left\langle\Phi(0) \Phi\left(r_{0}\right)\right\rangle}$ correlation function goes to zero, as $r$ approaches the event horizon. This leads us to the conclusion that the $O(2)$ symmetry of our model is not broken in the vacuum state of the static system, in other words the vacuum state of a geodesic observer.

\section{Discussion}

In Minkowski spacetime there is not much ambiguity about the concept of symmetry breaking. The first step, the choice of the coordinate system for the field quantization is natural, because the usual rectangular system has all possible attractive features: all coordinate lines are geodesics and translational invariance is manifest. The only symmetries which need more complex coordinate-transformations are the rotational symmetries and Lorentz boosts. However when we quantize the field, the vacuum state turns out to be also invariant under these symmetries. It is invariant even under the Lorentz boosts, although in the quantization process the equal-time hypersurfaces play a crucial role at the prescription of the commutation relations, and these hypersurfaces are certainly not invariant under Lorentz-boosts. Also, the n-particle states are mapped into n-particle states, only the particle 4-momenta transform correspondingly under the symmetries. Thus all inertial observers have the same vacuum, and corresponding multi-particle states, which can be used for studying symmetry breaking, and possible restoration processes of the symmetric vacuum state, for example in the case of finite volume.

As we move to a non-flat spacetime, these natural choices disappear. We have studied field theory in a de Sitter background, which has constant spacetime curvature, and a 10-element symmetry (like the Minkowski symmetry group, which is a contraction of the de Sitter group). But there is no coordinatization of the manifold that would consist of geodesics and would have translational invariance in all of its coordinate parameters. Furthermore there is no unique vacuum for all geodesic observers. We have to make our choices for equal-time surfaces, which will then affect the quantization through the commutator-prescriptions, that will lead to the corresponding vacuum states and Fock-spaces (for discussions of this aspect see for example [5] and [7]), and finally determine the existence or absence of symmetry-restoration processes (which are due to certain infrared divergences, therefore crucially depend on the properties of the Fock-space).

Since it is not only a possibility to arrive to different answers in different coordinate systems, but indeed the answer was found to be different using different systems previously $[1,4]$, we think that it is useful to study the problem through a system that is selected to correspond to the measurements of a geodesic observer, leading to a Fock-space where the vacuum state is the one in which the observer does not detect particles, etc. (see also [8] and [9]). The coordinate system that satisfies these requrements is the "static" system of the observer, in which the spatial sections are geodesic hypersurfaces orthogonal to the world line of the observer, with the spatial coordinates translated along the world line of the observer. 
As discussed in [1], the method we have employed searches for spontaneous symmetry breakdown by examining $\langle\Phi(0) \Phi(r)>$ as $r$ gets large. If this quantity tends to a positive constant, it would imply symmetry breaking. On the other hand if it tends to zero (which is what we have found), than that is consistent with the restoration of symmetry. It is still conceivable, however, that symmetry breaking does take place in this coordinate system, but manifests itself in a more subtle form. Studying the structure of the Fock-space might give more insight into this possibility, as well as into the physical reasons and the meaning of the different results that were obtained in refs. [1] and [4] on the subject of symmetry restoration studied in different coordinate systems of the same manifold. We intend to study the problem from this direction in a later paper.

\section{Acknowledgements}

We are grateful to Hisao Suzuki and Atsushi Higuchi for helpful discussions.

Research supported in part by DOE grant no. DE-AC02-ERO3075.

\section{References}

[1] B. Ratra, Phys. Rev. D 31, 1931 (1985).

[2] G. W. Gibbons and S. W. Hawking, Phys. Rev. D 15 , 2738 (1976).

[3] B. Allen, Nucl. Phys. B 226 , 228 (1983).

[4] B. Ratra, Phys. Rev. D 50 , 5252 (1994).

[5] N. D. Birrell and P. C. W. Davies, Quantum fields in curved space, Cambridge monographs on mathematical physics, 1985.

[6] A. Higuchi, Class. Quantum Grav. 4, 721 (1987); Haru-Tada Sato and Hisao Suzuki, OU-HET 202, LMU TPW 94-13, hep-th 9410092 (1994).

[7] E. Mottola, Phys. Rev. D 31,754 (1985).

[8] W. G. Unruh, Phys. Rev. D 14,870 (1976).

[9] A. Z. Capri and S. M. Roy, Int. Journ. of Mod. Phys. A 9 , 1239 (1994).

[10] M. Abramowitz and I. A. Stegun, Handbook of mathematical functions, Dover books on advanced mathematics, 1972. 